\title{
2D PHASE UNWRAPPING BY FUSION CUT-LINE AND QUALITY-MAPS
}

\author{
Faiza HOCINE, Sawssen BELHADJ-AISSA, Mohamed FEKIR, Aichouche BELHADJ-AISSA
}

\author{
University of Sciences and Technology Houari Boumediene (USTHB). Laboratory of Image Processing and Radiation, \\ Faculty of Electronic and Computer Science. \\ BP. 32, El Alia, Bab Ezzouar 16011, Algiers, Algeria,Email:faiza_ho@yahoo.fr, h.belhadj@lycos.com.
}

\begin{abstract}
Phase unwrapping is one of the critical step in the feasibility of InSAR processes. It consists in retrieving the absolute phase from modulo $2 \pi$. Through this paper, we propose a method of phase unwrapping that combines /fusions the advantages of two methods, namely: The method of phase unwrapping by avoidance of discontinuities, based on creating cut lines and the second method, it is based on local/regional propagation of phase unwrapping by referring to quality maps. The idea is that the quality value between the two target edges of the cut-line is set at zero in the quality maps, then the progress of the unwrapping is performed according to the process of the second method where unwrapping phase by referring to edge quality map. To validate the results, the method has been tested using real interferogram.
\end{abstract}

Keywords: InSAR, phase unwrapping, cut lines, quality maps, edge quality.

\section{INTRODUCTION}

The phase unwrapping consists at lifting the $2 \pi$ ambiguity in the phase and produce a continuous phase image proportional to the ground measurement, by seeking the integer multiple of $2 \pi$ for each pixel of the interferogram. In the ideal case where the phase difference between two adjacent pixels does not exceed $\pi$ (knowing that the Nyquist condition is met) [1], the unwrapping solution is the same regardless of the path of integration. Otherwise, such a problem has no unique solution. To this end, various phase unwrapping methods, techniques and algorithms have been proposed and developed [2] whose main idea is to minimize the phase differences between adjacent pixels: i) either, locally by correcting the phase step by step and imposing certain restrictions on the unwrapping path. [3] [4], ii) or, globally by modeling and optimization of possible solutions [5][6]. Through this paper, we propose a method of phase unwrapping that combines /fusions the advantages of two methods, namely: The method of phase unwrapping by avoidance of discontinuities, based on creating cut lines. It is extremely fast and generally satisfactory, is founded on residue localization and it proceeds to their effect neutralization to avoid them in the unwrapping step. They are created through connecting the positive and negative residues, either by straight lines or by lines following the low values of the coherence image. The disadvantage of this method is that it creates isolated areas "due to sudden cuts in the phase" and the second method, it is based on local/regional propagation of phase unwrapping by referring to quality maps. The quality of a pixel is calculated compared to all these neighbors, unlike other methods of calculation. The fact that each phase unwrapping operation is performed between two adjacent pixels, then instead to interested on the quality of isolated pixel, we look on the pixel edge quality, which is the intersection of two pixels connected horizontally, vertically or on the diagonally direction. Then the edge quality of the pixels is used to guide the path of unwrapping phase. However, this method has a tendency to create an effect of blocks in unwrapped phase space and in the presence of noise or discontinuities of phase, phase unwrapping is itself unresolved and hence quality-guided phase unwrapping is not sufficient. To improve results, our method creates the cut lines prohibiting the path of progress unwrapping along the path of quality calculated from the interferogram. To validate the results, the method has been tested using real interferogram product from tandem pair ERS1/ERS2 taken in the region of Algiers, Algeria.

\section{PHASE UNWRAPPING}

The concept of phase unwrapping consists to seek for a single reconstruction path of a curve or a continuous surface. It progresses through the calculation of the phase difference between two successive pixels in a predefined way. If the difference is less than or greater than $\mp \pi$, successive pixels in the path are shifted by $\pm 2 \pi$. The trivial process flow is summarized as follows:

$$
\begin{array}{ll}
\text { if } \mid \Delta \varphi\left(p_{1}, p_{2} \mid \leq \pi\right. & \text { then } \Delta \Phi\left(p_{1}, p_{2}\right)=\Delta \varphi\left(p_{1}, p_{2}\right) \\
\text { if } \Delta \varphi\left(p_{1}, p_{2}\right)>\pi & \text { then } \Delta \Phi\left(p_{1}, p_{2}\right)=\Delta \varphi\left(p_{1}, p_{2}\right)-2 \pi \text {, } \\
\text { if } \Delta \varphi\left(p_{1}, p_{2}\right)<-\pi & \text { then } \Delta \Phi\left(p_{1}, p_{2}\right)=\Delta \varphi\left(p_{1}, p_{2}\right)+2 \pi
\end{array}
$$

Where $p_{1}, p_{2}$ are two neighboring pixels, $p_{1}$ represent the reference point, $p_{2}$ is unwrapped according to $p_{1}, \Delta \varphi$ et $\Delta \Phi$ are the difference of the wrapped phase and the unwrapped phase respectively. As a result of this step we obtain a continuous phase as shown in the profile of a line of the unwrapped phase 'Figure 1'. This curve contains the information of the unwrapped phase in respect to the first pixel of the line. The coherence of the 
unwrapping results is to not have any phase jump from one pixel to another higher than $2 \pi$.

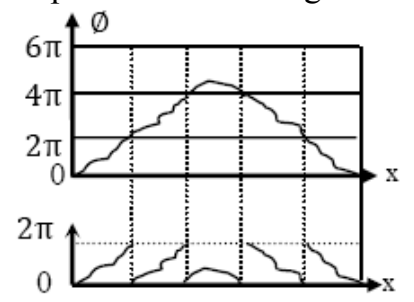

Figure 1. Principle of phase unwrapping.

This step of the interferometric process has been the main subject of several research studies using different global, local and regional approaches. In this paper, we propose a hybrid strategy which is based on two regional approaches.

\section{PROPOSED STRATEGY}

The proposed strategy combine two methods, which, in the first one the phase is unwrapped regardless of the chosen path [3], its principle is to unwrappe the phase without crossing the branch cut, those are created by associating two residues which quantify the phase discontinuities, in other words, this method concept is to avoid the error propagation by avoiding the phase discontinuities. The drawbacks is that isolated areas are created. Those, generate an important phase jumps around the Branch Cut while unwrapping the phase.

The second method rely on the quality of a pixel to define the unwrapping path [7]. The accuracy of this algorithm depends mainly on the quality map. However, the result obtained tend to bring up a block effect particularly in areas with low coherence.

In this sense, the main purpose of the proposed algorithm is to minimize the drawbacks of these two methods.

This algorithm is summarized by the diagram in the figure below:

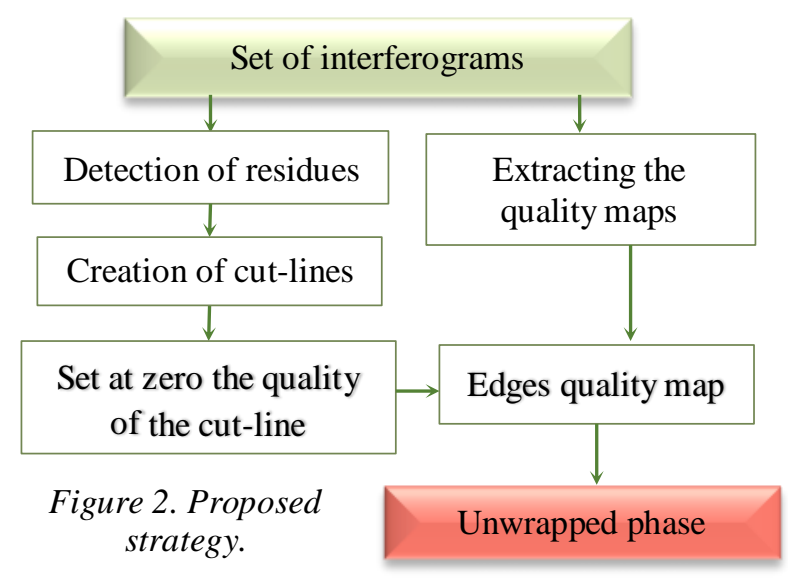

\section{PHASE UNWRAPPING AVOIDING BRANCH CUTS}

The interferometric phase presents some discontinuities that can cause error propagation during the unwrapping. These discontinuities are quantified and the result in an image of residues with positive and negative polarity.

The branch cut are then created by connecting two opposite polarities residues. While the unwrapping these cut should be avoided.

The basic method, in this approach, was developed by Goldstein et al in 1988 [3]. Its principle is to find the most appropriate way to unwrappe the phase by ensuring the coherence of the outcome. It is based on the following properties:

- No error propagation over a closed path if and only if the sum of the phase differences corrected along this path is zero.

- The closed unwrapping path must satisfy the following condition:

$$
\sum_{i=1}^{m} \gamma\left[\varphi_{i}-\varphi_{i+1}\right]=0
$$

However, this method creates areas trapped by Branch Cut and are inaccessible by the unwrapping algorithm. One solution to this problem has been proposed in [8], the algorithm called dipole Branch Cut ', is based on the same properties as the basic method (Goldstein algorithm). It uses the coordinates of the positive or negative residue to search for the closest opposite polarity residue and connect them to a unique Cut line (dipole line). However, these lines turn out to be too long, so an optimization techniques such as simulated annealing or the minimization of the cost function [9] [10] have been used to look for an optimal neighborhood.

Compared to the basic algorithm, the cuts produced by these algorithms are shorter with the absence of isolated zones especially in the noisy regions. Nevertheless, these algorithms can produce cuts in the wrong places, because two nearby residues do not necessarily belong to the same fringe, limited by the cut between them. Therefore, the solutions proposed by these researchers are neither unique nor optimal, but quick with consistent results especially when the density of the residues is low.

\section{PHASE UNWRAPPING GUIDED BY QUALITY MAP}

This approach was first proposed by Bone [11], it uses only quality map to set the best unwrapping path. It relies, not only on, local analysis and modeling of relations between neighboring pixels, but also, on a global evolution of the context through an iterative update of an unwrapped phases according to the neighborhood.

The methods based on this principle start the unwrapping from the pixel having the highest quality value to the pixel with the lowest one.

The accuracy of these procedures is mainly due to the way the quality map is calculated and the evolution of 
both intra and inter-fringe areas of the interferograms. Still, they are strongly dependent on the quality indicators. However, most of these are processed from the interferogram using image processing techniques without any knowledge on the phase discontinuities. Their development is based on two levels of treatment:

- Estimation of the phase's quality indicator

- Unwrapping the phase from the high quality region to the low quality region.

\subsection{Quality map}

In the proposed algorithm, we used a quality map calculated by the second difference.

The estimation of the second derivative of the phase in a neighborhood can reveal the abrupt transitions of the interferometric phase. This measurement is evaluated for each pixel in a window of size $\mathrm{k} * \mathrm{k}$ and calculated as follows:

$$
\mathrm{SD}_{m, n}=\frac{1}{\sqrt{\left(\Delta_{H}\left(\varphi_{m, n}\right)\right)^{2}+\left(\Delta_{V}\left(\varphi_{m, n}\right)\right)^{2}+\left(\Delta_{D 1}\left(\varphi_{m, n}\right)\right)^{2}+\left(\Delta_{D 2}\left(\varphi_{m, n}\right)\right)^{2}}}
$$

Avec $\mathrm{SD}_{m, n}=\Delta\left(\varphi_{m, n}\right)$

Où:

$\Delta_{H}\left(\varphi_{m, n}\right)=\gamma\left[\begin{array}{ll}\varphi_{m, n-1} & -\varphi_{m, n}\end{array}\right]-\gamma\left[\varphi_{m, n}-\varphi_{m, n+1}\right]$

$\Delta_{V}\left(\varphi_{m, n}=\gamma\left[\varphi_{m-1, n}-\varphi_{m, n}\right]-\gamma\left[\varphi_{m, n}-\varphi_{m+1, n}\right]\right.$

$\Delta_{D 1}\left(\varphi_{m, n}\right)=\gamma\left[\varphi_{m-1, n-1}-\varphi_{m, n}\right]-\gamma\left[\varphi_{m, n}-\varphi_{m+1, n+1}\right]$

$\Delta_{D 2}\left(\varphi_{m, n}\right)=\gamma\left[\varphi_{m+1, n}-\varphi_{m, n}\right]-\gamma\left[\varphi_{m, n}-\varphi_{m-1, n+1}\right]$

$\gamma($.$) is an operator for correcting the phase between the$ two pixels by adding / subtracting $\pi$ respectively whenever the difference is less than $\pi$ or higher than $-\pi$. In the basic algorithm the neighboring pixels are unwrapped in respect to the central pixel having a high quality indicator. Thus, a neighboring pixel is unwrapped even do it has a low quality indicator. As a solution to this problem, Herráez et al [7] considered, instead of a pixel quality, an edges quality, horizontal and vertical.

In this case, the unwrapping is proceed, at first, on two pixels that form the edge with the highest quality value. Then the two pixels of the second edge's highest quality value and so on.

\subsection{Unwrapping procces}

The processing steps can be summarized as follow: Initially all pixels are not unwrapped and do not belong to any group. During the process, the following situations are encountered:

$\checkmark$ If two pixels do not belong to any group, the two pixels are unwrapped one against the other and a new group is created.

$\checkmark$ If a pixel is already unwrapped, the not-unwrapped pixel is thus unwrapped against the already unwrapped one, and it is attached to the unwrapped pixel group.

$\checkmark$ If the two pixel are already unwrapped and do not belong to the same group, the unwrapping of all the pixels of the smallest group is done against the pixels of the other group.

\section{RESULTS AND DISCUSSIONS}

To evaluate the obtained results and the performance of the proposed algorithm, we conducted tests on a real interferogram 'Figure 3.a', extracted from a pair of tandem sensors ERS1/ERS2 acquired on $3^{\text {rd }}$ and $4^{\text {th }}$ January 1996, for Algiers area (Algeria). This interferogram corresponds to area of hilly terrain (see the fringes pattern in the lower part of Figure 3.a).

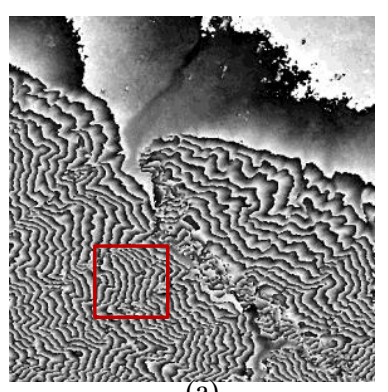

(a)

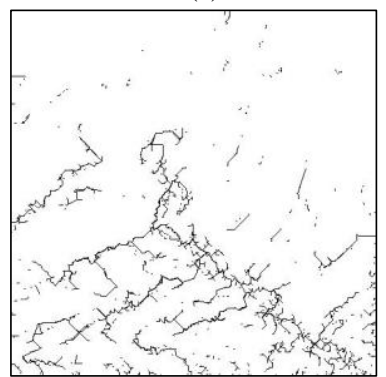

(c)

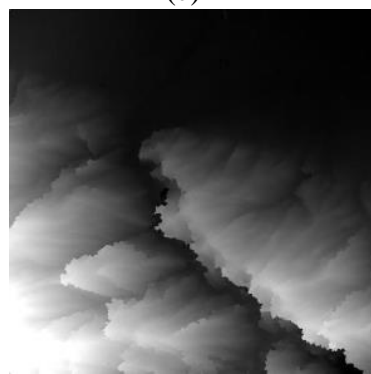

(e)

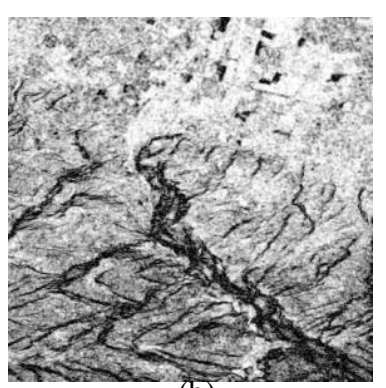

(b)

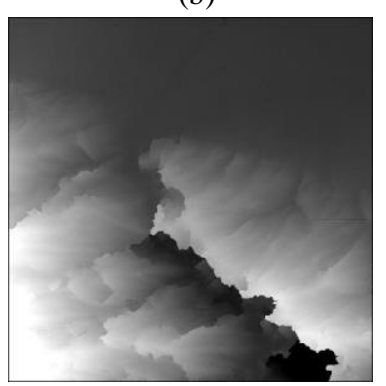

(d)

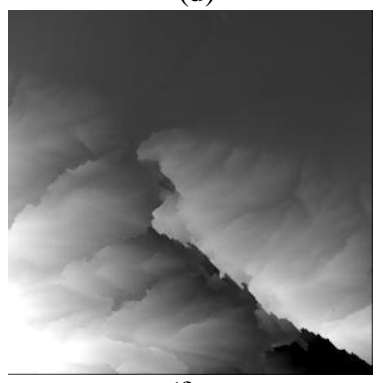

(f)
Figure3. Unwrapping phase of real interferogram.

a. real interferogram (phase wrapped),b. quality map, $c$ : brunch-cut line, $d$ : unwrapping using cut-brunch, $e$. unwrapping using quality map, $f$. unwrapping using proposed algorithm.

Figure. 3 shows that the conflict zones are in areas with low coherence.

The proposed method keeps the phase's jumps only in areas with a low coherence (see dotted arrows in Figure $4 \mathrm{~b}$, $\mathrm{f}$, which is an enlargement of a region from Figure $3)$. 
We also note that fig.4.c cut lines are positioned mainly in areas with low coherence. After the unwrapping process phase jumps are created (shown in fig.4.d arrows). This is due mainly to the cut lines that delineate the areas with low coherence.

Furthermore, the method based on the quality map shows a bloc effect due to the fact that the phase unwrapping process evolve region by region.

The inclusion of the cut lines' image as a parameter in the unwrapping process improved the results. As can be seen on fig.4.f.

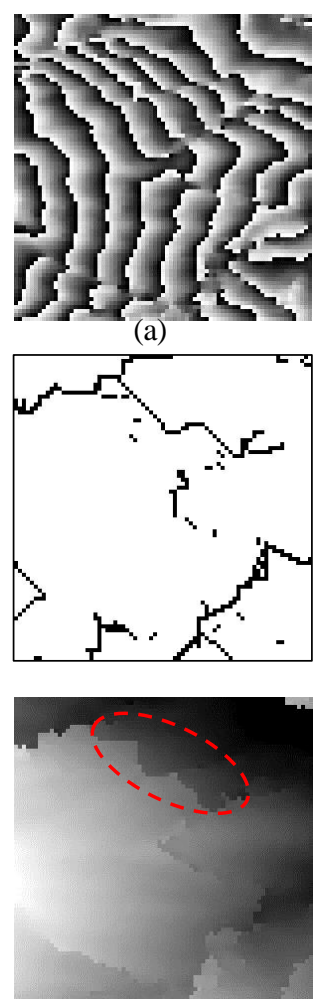

(e)

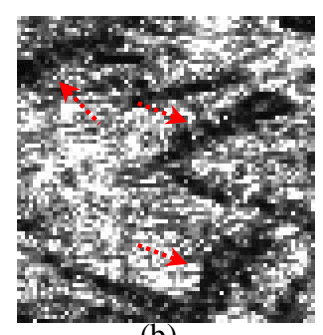

(b)
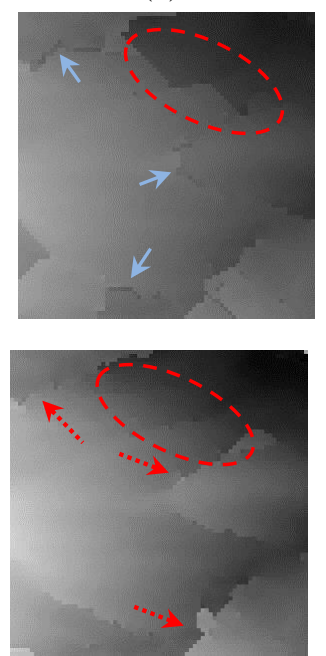

(f)
Figure 4. Unwrapping phase of real interferogram. a. real interferogram (phase wrapped), b. quality map, $c$ : brunch-cut line, $d$ : unwrapping using cut-brunch, e. unwrapping using quality map, f. unwrapping using proposed algorithm.

Both profiles of figure.5, can confirm the results. Add to that the proposed method has better unwrapped phase continuity.

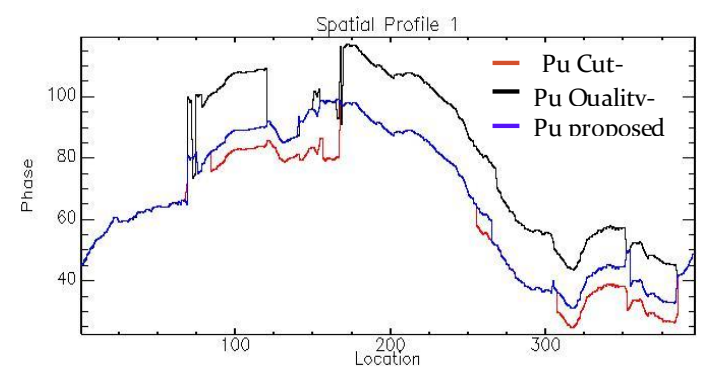

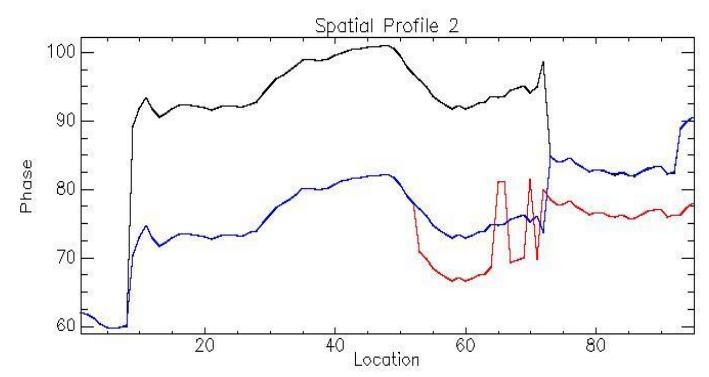

Figure 5. Phase profiles.

\section{Conclusion}

Although the problem of 2D phase unwrapping has been extensively studied and has been the main interest of various studies, particularly in SAR interferometry. The convergence towards an optimal solution reflecting the physical reality is far from being achieved. Different phase unwrapping methods have been developed. Where, each method has its specifications to its results. The proposed algorithm which is based on fusion cutline and quality-maps is allowed a good continuity of the unwrapped phase at the boundaries of the fringes.

\section{REFERENCES}

1. Bone, D. J. (1991). Fourier fringe analysis: The twodimensional phase unwrapping problem. Applied Optics. 30(25), 3627-3632.

2. Ghiglia, D. C., Pritt, M. D. (1998). Two-Dimensional Phase Unwrapping: Theory, Algorithms, and Software, New York: Wiley, pp4-178.

3. Goldstein, R. M., Zebker, H. A. \& Werner, C. L. (1988). Satellite radar interferometry: Twodimensional phase unwrapping. Radio Science, 23(4), 713-720.

4. Karout, S. A., Gdeisat, M. A., Burton, D. R., Lalor, M. J. (2007). Residue Vector a New Approach to Branch Cut Placement in Phase Unwrapping - the Theoretical Study. Applied Optics, 46.

5. Ferraioli, G., Shabou, A., Tupin, F., \& Pascazio, V. (2009). Multichannel phase unwrapping with Graph-cuts, IEEE Trans. Geosci. and Remote Sensing Letters, 6(3), 562-566

6. Valadão, G., Bioucas-Dias, J. (2009). CAPE: combinatorial absolute phase estimation, Optical Society of America, 26(9).

7. Herráez, M. A., Burton, D.R., Lalor, M.J. \& Gdeisat, M.A. (2002). Fast two-dimensional phaseunwrapping algorithm based on sorting by reliability following a noncontinuous path, Applied Optics, 41, 7437-7444.

8. Huntley, J. M., (1989), Noise-immune phase 
unwrapping algorithm, Applied Optics, 28(15), 3268-3270.

9. Cusack, R., Huntley, J. M. \& Goldstein, H. T. (1995). Improved noise-immune phase unwrapping algorithm, Applied Optics, 34(5), 781-789.

10. Prati, C., Giani, M. \& Leuratti, N. (1990). SAR interferometry : a 2D phase unwrapping technique based on phase and absolute values informations, In IGARSS'90, Washington D.C., USA, 3, pp. 2043-2046.

11. Bone, D. J. (1991). Fourier Fringe Analysis: The Two-Dimensional Phase Unwrapping Problem. Applied Optics, 30, 3627-32. 